\title{
MAKE IT MATTER: \\ How to foster interest in particle physics by setting it in meaningful contexts
}

\author{
Sarah Zöchling ${ }^{a, b, *}$, Martin Hopf ${ }^{a}$, Julia Woithe ${ }^{b}$, and Sascha Schmeling ${ }^{b}$ \\ a University of Vienna, AECC Physics \\ Porzellangasse 4/2/2, 1090 Vienna, Austria \\ b CERN, \\ Esplanade des Particules 1, 1211 Geneva 23, Switzerland \\ E-mail: sarah.zochling@cern.ch, martin.hopf@univie.ac.at, \\ julia.woithedcern.ch, sascha.schmeling@cern.ch
}

Research in particle physics seems to be abstract and far away from high-school students' daily life. Yet, research in particle physics is not only relevant for scientists but also applied in numerous fields. For example, technologies developed at CERN are used in medicine for cancer diagnostics and therapy or in cultural heritage for art authentication and restoring. These applications of particle physics may be interesting for high-school students, and thus could serve as contexts for learning activities about particle physics contents. In the framework of a $\mathrm{PhD}$ project in physics education research at CERN, a study examines how to foster students' interest in particle physics by setting it in meaningful contexts. The aim of the project is to compare different contexts in order to identify the ones that are equally and highly interesting for all students. We developed an instrument to measure particle physics interest (IPPI). The items present particle physics set in different contexts. We surveyed 1049 German-speaking students aged 14 to 15 years in an online cross-cohort study. Rasch analysis revealed which contexts of particle physics were rated as more (or less) interesting by the students. For example, the most interesting context was the human body ("medical diagnostics"). Knowing the hierarchy of interesting contexts enables educators to adapt or create learning activities according to the most promising contexts.

The European Physical Society Conference on High Energy Physics (EPS_HEP2021)

26-30 July 2021

Online conference, jointly organized by Universität Hamburg and the research center DESY 


\section{Introduction}

We conduct a physics education research project about students' interest in particle physics in cooperation of the University of Vienna and CERN. The overall aim of physics education research is to improve physics education by considering students' and teachers' perspectives, i.e. teaching and learning. The aim of this project is to examine how to foster high-school students' interest in particle physics by setting it in meaningful contexts. Different contexts are compared in order to identify the ones that are equally and highly interesting for all students. For example, applications of particle physics in medicine or in cultural heritage may be interesting for all students, and thus could serve as meaningful contexts for learning activities about particle physics.

\section{Research Framework}

High-school students' interest in physics correlates with their course and career choices [1]. In education research, "interest" is defined as the "subjective value attached to knowledge about an object" [2]. The theoretical construct "interest" involves emotional, value-related, and cognitive-epistemic components [2]. The development of students' interest can be described with the "4-phase model of interest development" [3]. This model explains how a persons' triggered situational interest develops into individual interest, a relatively stable personality trait [3]. In this model, the emotional component of interest prevails initially but the value-related and the cognitive-epistemic components become more important from phase to phase [3]. When investigating high-school students' interest in science, previous studies focused on interesting (a) contents (e.g., pump) and (b) contexts (e.g., heart). Previous studies agree that the context has a greater influence on students' interest than the content $[4,5]$. However, since students differ in their preferences, they have been categorised into different interest types [4]. Yet, there are contexts (e.g., the human body) which are extremely and equally interesting for all students [4].

\section{Research Interest}

Previous studies about students' interest did not include modern physics, such as particle physics. Thus, we conducted a cross-cohort study guided by two research questions.

RQ1: Which contexts of particle physics are more (or less) interesting for German-speaking students aged 14 to 15 years?

RQ2: Into which different levels of interest in particle physics can German-speaking students aged 14 to 15 years be categorised?

We hypothesised that everyday contexts, such as the human body or nature, are more interesting for high-school students than purely scientific contexts, such as qualitative physics. In addition, we hypothesised that students at a level of focused interest in particle physics are interested in particle physics solely when particle physics is set in an everyday context. Finally, we hypothesised that students at a level of broad interest in particle physics are additionally interested in particle physics when set in purely scientific contexts.

\section{Research Design}

The research setting of our project is "S'Cool LAB", an education laboratory on-site at CERN in Geneva, Switzerland. In S'Cool LAB, high-school students and teachers contribute to physics education research projects by taking part in different on-site or online learning activities. Typically, these activities are focused on a particular particle physics content set in different 
contexts. For example, the on-site experiment session about X-rays is currently set in the contexts "Medicine" (X-ray images) and "Technology" (airport security). In this project, we conducted a cross-cohort study to investigate high-school students' interest in particle physics utilising an online measurement instrument due to the COVID-19 pandemic. First, we developed the instrument to measure particle physics interest ("IPPI") based on the "IPN Interessensstudie Physik", student think-aloud interviews ( $\mathrm{N}=16$, April-May 2020), and a field test ( $\mathrm{N}=99$, June 2020) using Rasch theory and analysis [6]. The IPPI consists of an introduction text and 11 ratingscale items. Each item presents particle physics set in a different context. The students express their interest in each item on a 5-category rating scale ranging from "My interest in it is ..." very high (=5) to very low $(=1)$.

\section{Participants}

We sent out an e-mail invitation to participate in the study directly to several randomly selected physics teachers in Austria, Switzerland, and Germany as well as via one Austrian and one German mailing list (AT: "Plus Lucis", DE: "Netzwerk Teilchenwelt"). As a result, 1049 students aged 14 to 15 years from Austria ( $N=788)$, Switzerland ( $=180)$, and Germany $(\mathrm{N}=81)$ participated in the study from June to July 2021. Both sexes were represented equally in the sample (498 female, 475 male, 76 not specified).

\section{Analysis and Results}

We analysed the data collected in this study using the mixed-Rasch rating scale model. We compared the analysis results for three different models, from only one interest type (i.e. the sample is not divided into different interest types) to a categorisation into three interest types. We used the BIC value as a criterion for model validity. A model with a smaller BIC value matches the data better than other models with higher BIC values. We found that the BIC value of the 3 types-model is the smallest, i.e. the 3-types-model describes the data the best. The delta BIC values indicate strong evidence against the 1-type- and 2-types-models. Consequently, students of this sample can be categorised into three different interest types. Then, we examined the item hierarchy revealed by the Rasch analysis for the three interest types. The item hierarchy shows which items are more (or less) interesting. We found that the item hierarchy for the interest types 1 and 2 is identical, yet different from interest type 3 . Analysis of the usage of rating scale categories for interest types 1 and 2 revealed that type- 1 students barely use the extreme values of the rating scale, while type- 2 students often use extreme values. Thus, type- 1 and type- 2 students only differ in their category usage but not in their item hierarchy. In the following, we analyse this item hierarchy that is valid for $75 \%$ of students (interest types 1 and 2) to define and characterise students' levels of interest as shown in figure 1. Analysis of this item hierarchy revealed that the most interesting items present particle physics set in contexts related to one's own body, socio-scientific issues, or existential questions of humankind. We argue that these contexts trigger emotions and that students at the hypothesised level of focused interest are only interested in these contexts. Items that present particle physics set in everyday life contexts using specific examples were rated as less interesting. We argue that these contexts emphasize the personal value. Based on this finding we introduce the level of open interest. Students at the level of open interest are additionally interested in these everyday life contexts. The least interesting items present particle physics set in contexts related to science and technology. We argue that only 
students at the hypothesised level of broad interest are interested in particle physics set in these cognitive-epistemic contexts. This characterisation of levels of interest aligns with the hierarchy of interest components (emotional, value-related, cognitive-epistemic) described in the "4-phase model of interest development" [3].

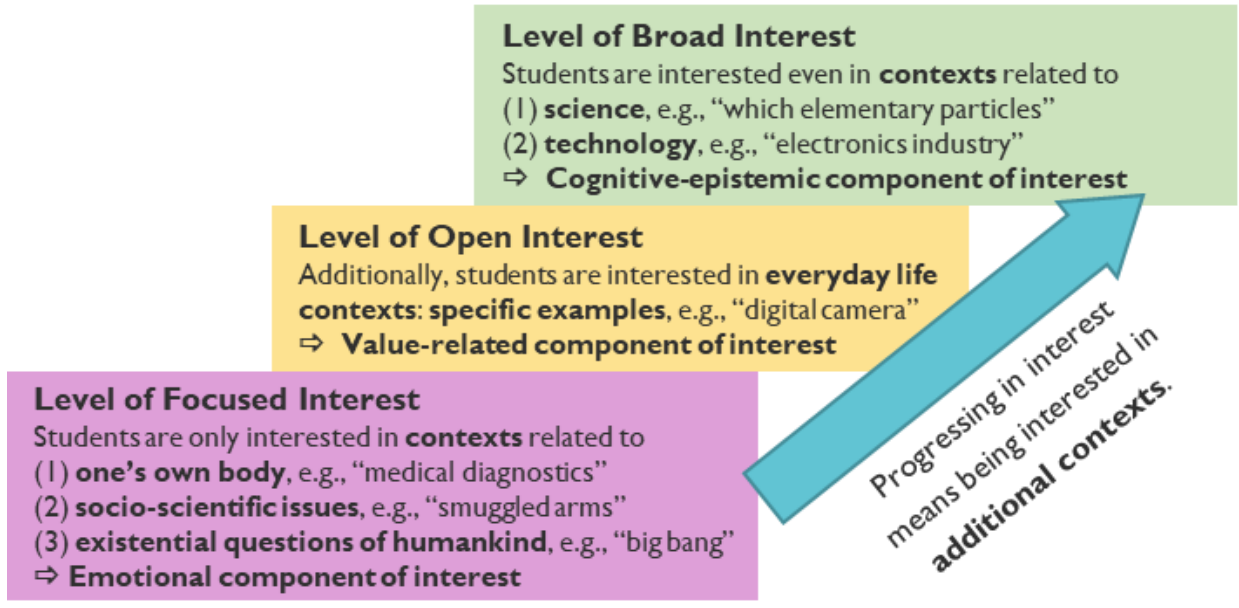

Figure 1: Characterisation of three levels of interest in particle physics that is valid for $75 \%$ of students and based on the item hierarchy revealed by the Rasch analysis

\section{Discussion and Next Steps}

Our study revealed that one item hierarchy is valid for $75 \%$ of students. Regarding our research questions, we found (1) that items are rated as more (or less) interesting depending on the context and (2) that students can be categorized into three levels of interest in particle physics, each associated with certain contexts, and students are interested in additional context as they progress through these levels of interest in particle physics. Our next steps are (1) to evaluate characteristics of students at the different levels, e.g. physics identity, and (2) to compare our findings for interest in particle physics to classical physics contents, in particular mechanics.

\section{Implications for Practice}

Educators trying to increase students' interests can match the design of their learning activities with students' different levels of interest. Students at all levels of interest are interested in particle physics set in contexts related to one's own body, socio-scientific issues, or existential questions of humankind. Students at the levels of open and broad interest are additionally interested in particle physics set in everyday life contexts using specific examples. Only students at the level of broad interest are interested in particle physics set in purely scientific contexts.

\section{References}

[1] Blankenburg, J. S., \& Scheersoi, A. (2018). Interesse und Interessenentwicklung. in Theorien in der naturwissenschaftsdidaktischen Forschung (245-259). https://doi.org/10.1007/978-3-662-56320-5 15

[2] Krapp, A., \& Prenzel, M. (2011). Research on Interest in Science: Theories, methods, and findings. International journal of science education, 33(1), 27-50. https://doi.org/10.1080/09500693.2010.518645

[3] Hidi, S., \& Renninger, K. A. (2006). The Four-Phase Model of Interest Development. Educational Psychologist, 41(2), 111-127. https://doi.org/10.1207/s15326985ep4102 4

[4] Häußler, P., Lehrke, M., \& Hoffmann, L. (1998). Die IPN-Interessenstudie Physik. IPN.

[5] Bennett, J., Lubben, F., \& Hogarth, S. (2007). Bringing science to life: A synthesis of the research evidence on the effects of context-based and STS approaches to science teaching. Science Education, 91(3), 347-370. https://doi.org/10.1002/sce.20186

[6] Zöchling, S., Hopf, M., Woithe, J. \& Schmeling, S. (2020). Spreading interest in particle physics among highschool students - what matters? PoS (ICHEP2020), 964, 1-6. 